\title{
A QoS-based Resources Reservation Mechanism for Ad Hoc Networks
}

\author{
Hassene Bouhouche \\ High Institute of Computing Mahdia ISIMa, \\ University of Monastir, Tunisia \\ SecNum Laboratory Research, SUP'COM
}

\author{
Sihem Guemara El Fatmi \\ High School of Communication SUP'COM, \\ University of the 7th of November at Carthage, Tunisia \\ SecNum Laboratory Research, SUP'COM
}

\begin{abstract}
For the last several years, there has been a significant increase of interest in supporting quality of service (QoS) constraints in Mobile Ad Hoc Networks (AHNs). AHNs include mobile nodes with limited capacities and communication resources. This specificity makes existing solutions for wired networks little suitable and a broad range of novel approaches have been studied. In this paper we propose a QoS reservation mechanism for AHNs, called QSRR. The mechanism is targeted for sources requiring a bandwidth allocation. It is based on the knowledge of the bandwidth requirements of the neighbours of a node and the interfering nodes in the cover area of each node. Our proposition uses a traffic classification and requires available bandwidth estimation definition. The advantages of this proposition are shown thanks to some simulation results that are detailed in the end of this paper.
\end{abstract}

\section{General Terms}

resources reservation mechanism, quality of service, Ad Hoc networks (MANET's)

\section{Keywords}

QoS resources reservation mechanism, Ad Hoc networks, QSRR, bandwidth allocation

\section{INTRODUCTION}

With the advancement of mobile devices, mobile applications and the deployment of AHNs in emergency rescue and military battlefields, the need to support real time communication or multimedia communication (voice, video) over AHNs have increased. These types of communication require QoS constraints like minimum bandwidths, maximum end-to-end delays, tolerable jitters...

QoS provisioning in AHNs is a challenging task. A AHN is a very complex distributed network where the nodes move in a free way making the topology of the network dynamic. Nodes moving do not only change the topology of the network but also cause dynamic changes in the traffic load. Moreover a node can act simultaneously as a source, a destination or an intermediate node. Others AHNs characteristics defy the QoS constraints that can be required by applications. One can mention bandwidth capacity and mobile device limitations like battery power and processing power.

Again, QoS provisioning should not put much load on the nodes and should not increase the volume of information to be maintained to support QoS in AHNs.

The elaboration of an adequate resource allocation mechanism in AHNs must take into account the mobility of the nodes and their characteristics and those of the physical medium. The complexity of such aspects is greatly increased when the quality of service (QoS) required by the AHN users is considered.

This paper is organized as follows: the Ad Hoc mobile environment's is very particular and the AHNs unit's constraints are very strong, we think it right to study in the second section of this paper these particularities. This section also deals with the concept of QoS in AHNs and details the classes of traffic (Real Time/ Best Effort). The third section of this paper defines the process of the available bandwidth estimation and proposes functions which quantitatively define the available bandwidth quantities. Section 4, exposes a novel proposition of resources reservation mechanism, called QSRR that takes into account the QoS challenge by defining a relation between the required constraints of the applications. In section 5, we expose the simulation results. Section 6 concludes this paper.

\section{QUALITY OF SERVICE IN AD HOC NETWORKS}

In this section we study the Ad Hoc networks environment's and the mobile unit's characteristics, the concepts of QoS in AHNS and finally we details the classes of traffic.

\subsection{The Ad Hoc Networks characteristics}

AHNs started with the aim to have the ability to establish a network among willing nodes without the assistance from any network infrastructure. AHNs are defined as a 'collection of mobile entities interconnected by a wireless technology forming a temporary network without the assistance of any administration or any fixed support where no centralized administration is available' [1]. Contrary to a cellular network, they are the mobile hosts themselves which form, in an ad hoc way, the network infrastructure.

This ability to establish this type of network seems to be very promising in situations like disaster or war (where the infrastructure is damaged or not available) or in areas where building the infrastructure is not possible or situations where an $\mathrm{AHN}$ is more suitable than an infrastructure network.

AHNs, which are based on IEEE 802.11 standard, are characterized by several limits and constraints [2] such as limited and shared bandwidth, limited energy, poor security, and so on. These characteristics make big differences between AHNs functioning and the one of traditional wired networks. In fact, dynamic topology and nodes arbitrary mobility can aggravate these difficulties especially because of the fast movements of the nodes and the variable conditions of the network which make the information of the network's state, obsolete quickly. 
Because of these characteristics, AHNs suffer of several problems such as the routing, the management of the mobility, the security, the quality of service and essentially what most interests us, the resources reservation. In fact, the resources reservation process constitutes one of the more illustrative and the more heavy examples of such differences between AHNs functioning and the one of traditional wired networks.

\subsection{Quality of Service in Ad Hoc Networks}

The QoS provided by a network constitutes nowadays an important issue for advanced applications because it concerns the different needs and constraints characterizing these applications. From the part of the network, it indicates its capacity to transport, under good conditions, flows resulting from various applications and various users [3]. Generally, it expresses a measure of the level of service that a particular data gets in the network. The basic idea behind 'provided' service is to differentiate between traffic coming into the network and provide preferential treatment to some types of data (Real Time applications). QoS also expresses a measure of performance and/or user satisfaction relative to a transmission system that reflects its transmission quality and availability of service.

The QoS can be defined as the manner that the service of delivery of packages is supplied and who can be characterized by various parameters of performance like the availability, the rate of errors, the response time, the delay, the throughput, the delay variation (jitter), the packet loss etc [4].

Unlike fixed networks, quality of service (QoS) support in AHNs depends not only on the available resources in the network but also on the mobility rate of such resources. This is because mobility may result in link failure which in turn may result in a broken path. Furthermore, AHNs potentially have less resource than fixed networks. Therefore, more criterions are required in order to capture the quality of the links between nodes.

We believe for AHNs, with time-varying low-capacity resources, the notion of being able to guarantee hard QoS is not plausible. Instead, applications must adapt to time-varying low-capacity resources offered by the network. Therefore, the QoS that an application requires depends on the "quality" of the network. This "quality" should be a function of available resources resides both in the wireless medium and in the mobile nodes in the network as well as the stability of such resources. Hence, QoS in AHNs could mean to provide a set of parameters in order to adapt the applications to the "quality" of network while routing them through the network.

Several architectures and techniques for the QoS provision have been defined and have been adopted for several types of networks. These techniques and architecture can not been applied in an AHN environment because of the particular characteristics of such networks such as the host functioning and the limited resources availability.

Providing QoS in AHNs has its own challenges and problems. For the QoS in the AHNs, some major networks aspects characterize a QoS expressed by a user or delivered by a network [5]. They are (1) the delay, (2) the resource availability and/or capacity, (3) the reliability, the bandwidth (4) and the rate of errors (5). We still speak about 'Capacity of the network'.

\subsection{Traffic classes}

We have considered three classes of traffic according to the applications QoS requirements. One on these classes has no QoS constraints [6]. The two others have strong temporal constraints and one of them has in addition strong bandwidth constraints. The three classes can be detailed as follows:

- Class 0: Real Time traffic Delay sensitive (CBR) generated by applications having strong temporal constraints: each bandwidth allowing the requested delay is acceptable by such traffic class. This class represents for example multimedia applications (audio).

- Class 1: Real Time traffic Bandwidth sensitive (VBR_RT) generated by applications having strong temporal constraints in addition to strong constraints in terms of bandwidth. A traffic generated by a video on demand application can be represented by a class 1 .

- Class 2: Best Effort Traffic (BE) generated by applications having no QoS constraints. For example a Web surfing application generated traffic of class 2 .

\section{AVAILABLE BANDWIDTH ESTIMATION}

In AHNs, available bandwidth in each node depends both of his consumption, the consumption of all his direct neighbours and the interferences caused by all these transmissions. Otherwise, one application can not optimize its transmission without having a precise idea of the complete state of his neighbourhood in term of resources consumption.

The available bandwidth estimation is a fundamental operation for a QoS offer [7]. This operation is very difficult because of the approximate acknowledge of the network state and the random mobility of nodes.

For AHNs, this mechanism is generally placed in the MAC layer to allow the source to estimate the available bandwidth quantities. This estimation must to take into account node's mobility, interferences caused by the different transmissions and the hidden stations problem. The available bandwidth quantities must be permanently up to date especially after a congestion establishment or a reception of a duplicate acquittement (DUPACK).

Available bandwidth can be defined as the maximum throughput with which we can transmit (between two nodes) without interrupt flows transmitted on the Ad Hoc networks. This term must not to be confused with the 'link capacity' representing the maximum throughput which can attempt on this link, or with the 'unusable link capacity'. Knowledge of the available bandwidth quantity is required for admission control, QoS based routing, flow management and resources reservation [8].

Quantitatively, we define in our mechanism, QSRR, the available bandwidth quantity as:

-Let assume that BW (in bps) is the total bandwidth quantity on a node. The maximum available bandwidth quantity on a node can be defined by this function (1):

$$
\text { MAB (i) }=\mathbf{B W}(\mathbf{i})-\mathbf{x}(\mathbf{i})-\mathrm{SUM}_{\mathbf{j} \epsilon \mathrm{Ni}} \mathbf{x}(\mathbf{j})
$$

Where: $\mathrm{BW}(\mathrm{i})=$ Total bandwidth on the node $\mathrm{i}$,

$\mathrm{x}$ (i) = Used Bandwidth on the node $\mathrm{i}$,

$x(j)=$ Used Bandwidth by the node $\mathrm{j}$ neighbour of the node $\mathrm{i}$,

Node $\mathrm{j}=$ neighbour of node $\mathrm{i}$ and $\mathrm{Ni}=$ set of node $\mathrm{i}$.

- On a link (i, j), the available bandwidth is expressed by the following expression (2): 


$$
A B(\mathbf{i}, \mathbf{j})=\operatorname{MIN}\{\text { MAB (i), MAB (j) }\}
$$

- For a path $\mathrm{p}=(\mathrm{S}, \mathrm{i}, \mathrm{j} \ldots \mathrm{k}, \mathrm{D})$, where $\mathrm{S}$ : Source, D: Destination and $\mathrm{i}, \mathrm{j} \ldots \mathrm{k}$ the intermediate nodes; we have the Maximum Available Bandwidth guaranteed on the path is done by the formula (3):

$\operatorname{MAB}(\mathbf{p})=\operatorname{MIN}\{\operatorname{MAB}(S, i), \operatorname{MAB}(i, j), \ldots, \operatorname{MAB}(k, D)\}$

\section{RESOURCES RESERVATION MECHANISM}

AHNs resources reservation is a challenging task due to the lack of resources both in the wireless medium and in the mobile nodes as well as the frequent changes in network topology. As a result, resources reservation in such networks is more difficult than in wired networks. Moreover, in AHNs, it is essential to consider the quality of links and to take into account the time-varying topology and time-varying network resources.

An important problem associated with resources reservation in AHNs is to employ methods that ensure the adequate QoS for the applications. The running of a service through an AHN will be interrupted, if an intermediate node belonging to the path moves out of range during data transfers. This interruption requires a subsequent path re-discovery between the source and the destination and invokes some path-maintenance algorithm that eventually increases the end-to-end delay. For instance, it is possible that a path that was earlier found to satisfy some QoS requirements no longer does so due to the dynamic nature of the topology. In such case, it is important that the network intelligently adapts the session to its new and changed conditions.

The goal of our QoS resources reservation method, QSRR, is two-folds: first reserving network path that have sufficient resources to satisfy the QoS requirements of all admitted connections and second achieving global efficiency in resource utilization. For each flow on which we have provided some QoS guarantee, QSRR will allocate some resources which will be exclusively for its use. This will ensure that as soon as the packet of that particular flow comes, it will not have to wait for some path or resource to be freed and it will be transmitted to the next node instantly.

We notice that a particular resource allocated for a particular flow can be done in two ways:

- In-Band Signalling: the signalling information is piggybacked on the data packet. i.e. the current data packet will carry information about the next packet.

- Out-of-Band Signalling: the signalling information is exchanged through a separate channel between the nodes. This consumes more bandwidth and leads to an increase in network traffic.

Thus, In-Band Signalling is more adapted for AHNs in general and QSRR particularly.

During the rest of this section, we are going to focus on the characteristics of the resources reservation mechanism QSRR. We will first interest to the reservation request's types, then to the resources reservation's strategies and finally to the expression of a relation between the required constraints in term of delay and bandwidth in order to express the applications requirements.

\subsection{Ressources Reservation}

\subsubsection{One-Pass Vs Two-Pass}

There are tow types of resources reservation request:

- One-Pass: This type of reservation requires the use of a QoS routing protocol allowing obtaining paths satisfying the application's requirements (in term of delay, throughput and the number of jumps). In this case, the resources reservation is made during the first and unique passage.

- Two-Pass: This request / response return the minimal value of the available bandwidth along a given path by means of the use of two messages of control. This implies the fact that we need more signalling, that the used links must be bidirectional (it is not always still the case when we work within the AHNs) and that paths do not have to change between the passage of the request and the one of the response (however mobility is the first characteristics of AHNs).

For our mechanism, QSRR, it seems more adequate to work with the One-Pass's reservation request which allows a gain on delay. This factor (delay) is very important for the Real Time applications in a general manner and for the CBR applications particularly. Moreover this type of reservation allows us resolving some inconveniences of the Two-Pass's reservation such as the necessity of having bidirectional links or the change of path due to the mobility.

\subsubsection{Hard State Vs Soft State}

There are tow strategies of resources reservation:

- Hard State: Once the reservation is made, the resources stay in the flow's disposal until the source receives an acknowledgement of receipt (Ack) from the destination. This implies a waste of time and a wasting of the network resources especially in the case of a break of link which engenders the fact that even the message indicating this cut cannot be sent.

- Soft State: When the reservation is made, a timer is activated and will be updated in a permanent manner. In this way the reservation will have a lifetime. When the timer is sold, and even if we have not yet received an acknowledgement of receipt from the destination, the reserved resources are going to be released and can be so used for the other flows.

The resources reservation with the Hard State method is the simplest because it requires less path marking for the update of the state of the reservations, besides, it is not limited in the time.

As even, it seems to us, within the AHNs, that the Soft State's reservation is the most interesting to be integrated in QSRR because the links radio are unstable and can be broken at any moment. Thus, the use of this method of reservation (Soft State) allows a better optimization of the use of the available resources.

\subsection{Delay and Bandwidth Computing}

QoS-adaptation provides an interface for applications to submit their requirements. Some applications are capable to expand their QoS parameters, so that instead of being a single value indicating the constraints (in term of delay or throughput) needed by an application; it becomes a range of service classes in which the application can operate, together with the current reserved value within that range. This provides the network flexibility so that reservations can be maintained as network conditions change. Applications request QoS by specifying the minimum level of service they are willing to accept and the maximum level of service they are able to utilize, and then adapt to the specified 
point within this range that the network commits to provide, which may change with time. Changes in allocation have to be signalled to the application, which adapts its behaviour to match to what is available.

To offer bandwidth guaranteed QoS, the residual end-to-end bandwidth must be known. In wired networks this is a trivial task since the underlying medium is a dedicated point-to point link with fixed capability. However, in wireless networks the radio channel of every node is shared with all its neighbours. Due to the shared medium, a node can successfully use the channel only when all its neighbours do not transmit and receive packets simultaneously [9].

While the resources reservation is based on the available bandwidth, we use, in QSRR, an efficient method to obtain a relation between the required delay and the required bandwidth, as expressed in formula (4) and (5):

$$
\begin{gathered}
\text { D req }=\frac{B \text { req }}{\text { B max }-(B \text { res }+ \text { B req })} \\
\text { B req }=\frac{\text { D req (B max }- \text { B res })}{1+D \text { req }}
\end{gathered}
$$

Where:

- $D$ req: is the requested delay;

- $B$ req: is the requested bandwidth

- B max: is the maximum bandwidth supported by a link, e.g.: 2 Mbps, 11 Mbps, or 54 Mbps;

- B res: is the residual (unused) bandwidth

\section{SIMULATION RESULTS}

Once the proposed QoS-based resources reservation method's concepts have been completely defined, it becomes necessary to check their feasibility and evaluate their benefits. Simulation analysis is used here to evaluate the proposed scheme.

The goal of this section is to present the main aspects of the simulation model and its results.

\subsection{Simulations Environnement}

The simulation software is OPNET Modeler 14.5.

Two categories of parameters have been considered in our study: the input parameters and the output parameters. These parameters are as follows:

Input parameters: These include:

MANET Network of dimension $100 \mathrm{~m} * 100 \mathrm{~m}$.

Tow types of applications: CBR \& VBR_RT.

Tow types of Resources Reservation Mechanism: CSMA and QSRR.

- A number of nodes constituting our network: This number is variable between 10 and 50 (interval of 5 nodes).

Output parameters: These include:

Delay (sec),

Throughput (packets/sec), and,

Load (bps)

\subsection{Simulations Scenarios}

For the applications type, we use: - Only CBR applications, or,
- Only VBR_RT applications, or,

- Both CBR and VBR_RT applications.

For the Resources Reservation Mechanism, we use:

- CSMA protocol, or,

- Our QSRR mechanism.

The node number is varying between 10 and 50 (adding 5 nodes each time: $10,15,20,25,30,35,40,45$ and 50).

\subsection{Simulations Results}

In this sub-section, we try to show that the performances provided by our resources reservation mechanism, QSRR, are always better than the ones provided by the CSMA protocol. Theses performances are expressed in terms of delay, load and throughput.

\subsubsection{Comparison in term of delay}

The delay (expressed in $\mathrm{sec}$ ) represents the End to End (E2E) delay of all the data packets that are successfully received by each access category of the Wireless LAN MAC and forwarded to the higher layer. For the sake of this statistic, it is assumed that a packet is received by the same access category that is used for the transmission of the packet at the source node. This assumption is needed because access categories have no role within the MAC during the reception, processing and forwarding of the packets arrived from the physical layer.

The delay includes queuing and medium access delays at the source MAC, reception of all the fragments individually, and the relay of the frame via AP, of the source and destination MACs are non AP MACs of the some infrastructure BSS.

In a general manner, we notice that the delay provided by our resources reservation mechanism QSRR, is always lower than the one provided by CSMA, as shown in Figure 1, Figure 2 and Figure 3, and this whatever the distribution of the applications (\% of CBR and \% of VBR_RT applications) and whatever the number of nodes constituting our network.

For a given application distribution's, we notice that the delay increases with the increase of the number of nodes constituting the network, but the delay provided by QSRR remains always lower than the one provided by CSMA.

The Figure 1, Figure 2 and Figure 3 represent the QSRR and CSMA delay's evolution respectively for the application distribution 100\%CBR and 0\%VBR_RT, 50\%CBR and $50 \%$ VBR_RT and finally $0 \%$ CBR and $100 \%$ VBR_RT.

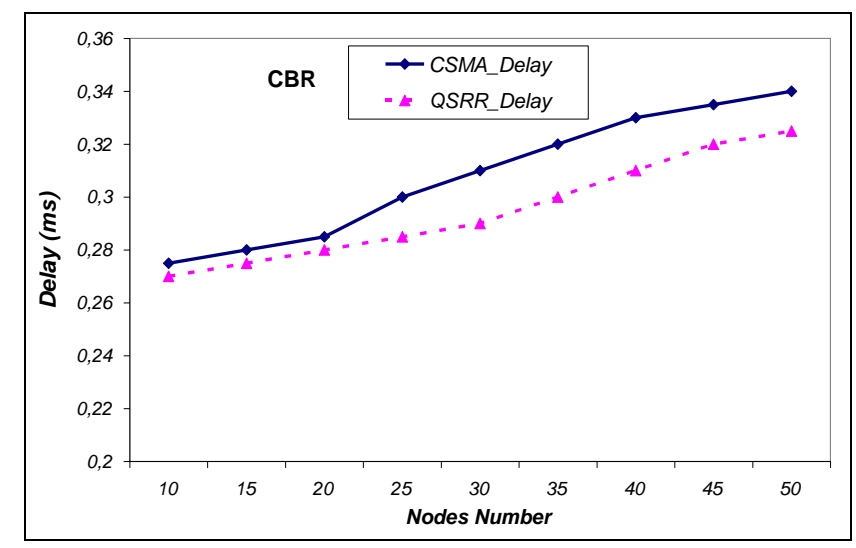

Figure 1. Delay evolution for applications 100\% CBR \& $0 \%$ VBR_RT 


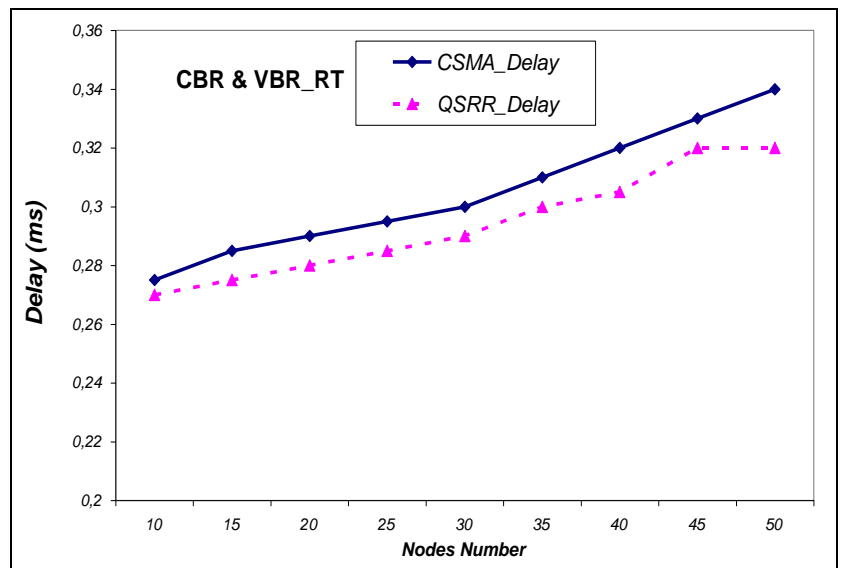

Figure 2. Delay evolution for applications 50\% CBR \& 50\% VBR_RT

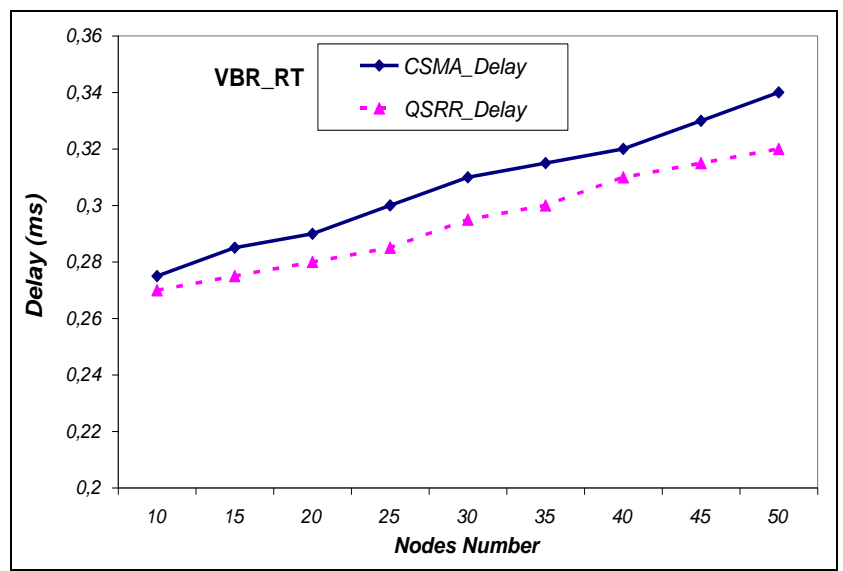

Figure 3. Delay evolution for applications 0\% CBR \& 100\% VBR_RT

\subsubsection{Comparison in term of Load}

The load (expressed in bit/sec) represents all data traffic received (in bps) by the Wireless LAN MAC from its higher layer for each of its access categories. Higher layer data packets are assigned to the access categories based on their user priority (class of service) values.

In a general manner, we notice that the load provided by QSRR, is always higher than the one provided by CSMA, as shown in Figure 4, Figure 5 and Figure 6 , and this whatever the distribution of the applications (\% of CBR and \% of VBR_RT applications) and whatever the number of nodes constituting our network.

For a given application distribution's, we notice that the load increases with the increase of the number of nodes constituting the network, but the load provided by QSRR remains always lower than the one provided by CSMA.

The Figure 4, Figure 5 and Figure 6 represent the QSRR and CSMA load's evolution respectively for the application distribution 100\%CBR and 0\%VBR_RT, 50\%CBR and 50\%VBR_RT and finally 0\% CBR and 100\% VBR_RT.

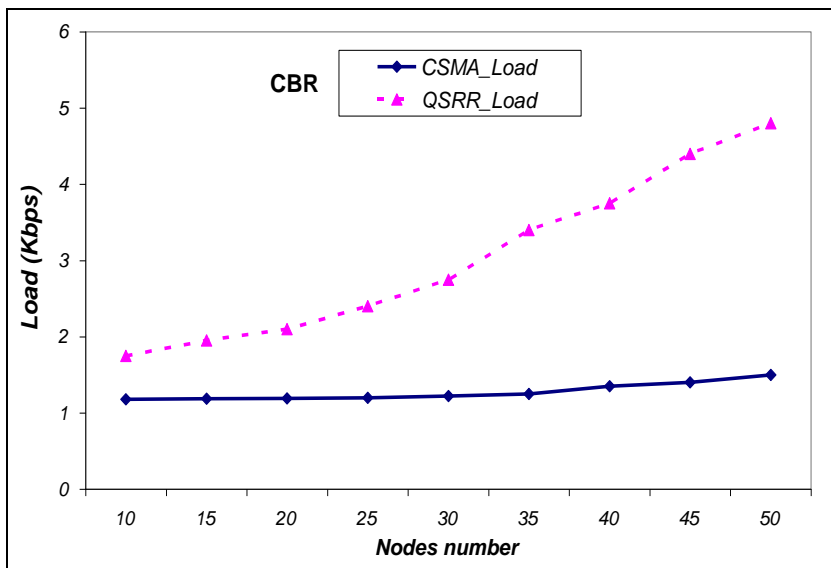

Figure 4. Load evolution for applications 100\% CBR \& $0 \%$ VBR_RT

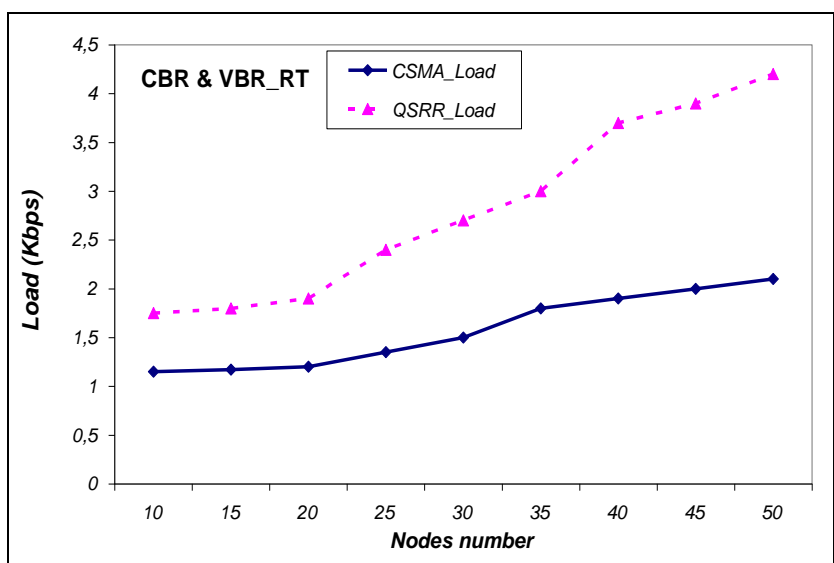

Figure 5. Load evolution for applications 50\% CBR \& 50\% VBR_RT

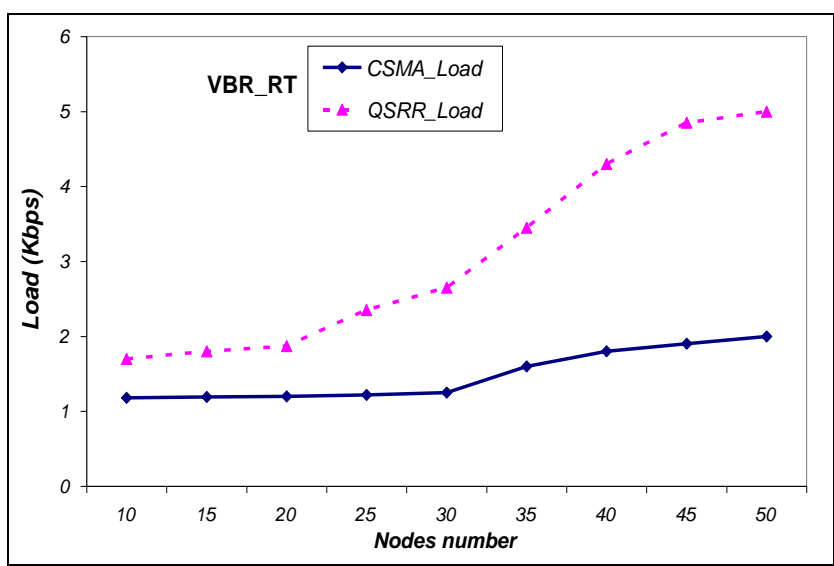

Figure 6. Load evolution for applications 0\% CBR \& $100 \%$ VBR_RT

\subsubsection{Comparison in term of Throughput}

The throughput (expressed in bit/sec) represents the total data traffic in bit per second successfully received and forwarded to the higher layer by each access category of the Wireless LAN MAC.

In a general manner, we notice that the throughput provided by QSRR mechanism, is always higher than the one provided by the CSMA mechanism, as shown in Figure 7, Figure 8 and Figure 9, and this whatever the distribution of the applications (\% of CBR 
and \% of VBR_RT applications) and whatever the number of nodes constituting our network.

For a given application distribution's, we notice that the throughput increases with the increase of the number of nodes constituting the network, but the throughput provided by QSRR remains always higher than the one provided by CSMA.

The Figure 7, Figure 8 and Figure 9 represent the QSRR and CSMA throughput's evolution respectively for the application distribution 100\%CBR and 0\%VBR_RT, 50\%CBR and $50 \%$ VBR_RT and finally $0 \%$ CBR and $100 \%$ VBR_RT.

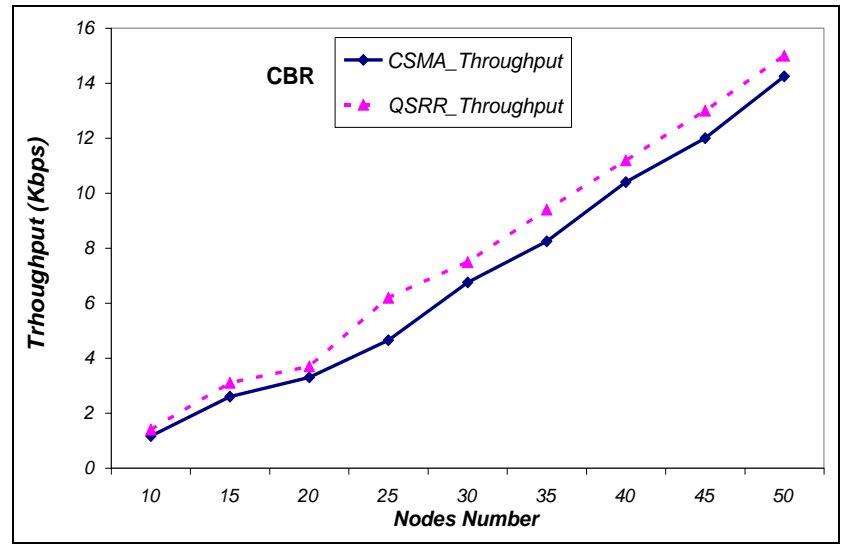

Figure 7. Throughput evolution for applications $100 \%$ CBR \& $0 \%$ VBR_RT

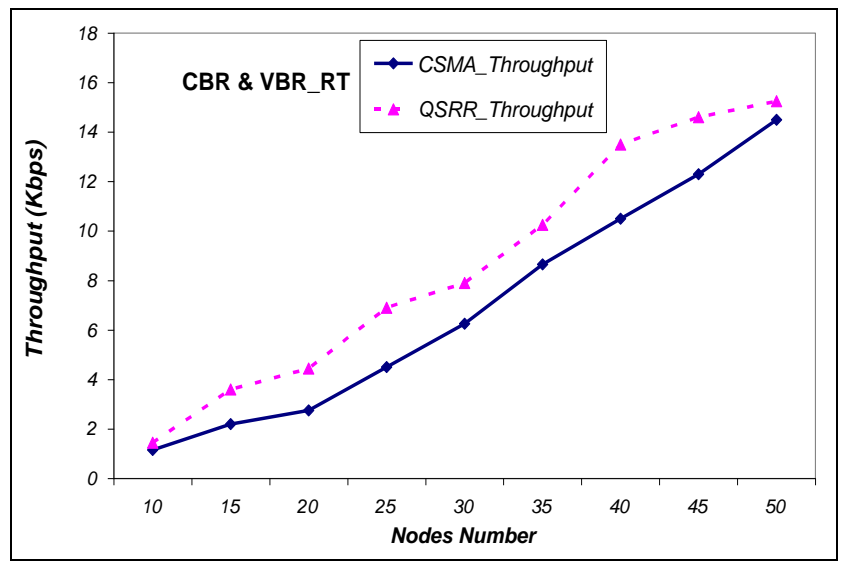

Figure 8. Throughput evolution for applications 50\% CBR \& 50\% VBR_RT

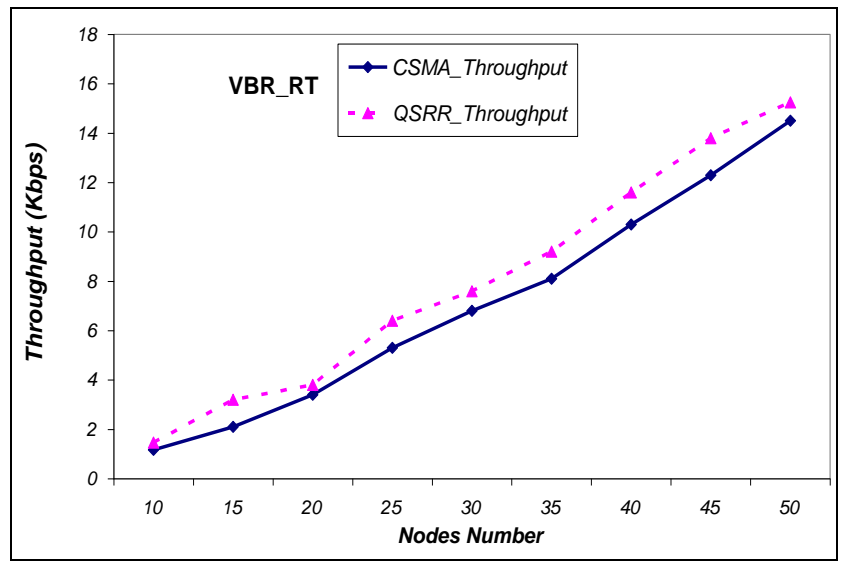

Figure 9. Throughput evolution for applications $0 \% \mathrm{CBR} \&$ $100 \%$ VBR_RT

\section{CONCLUSION}

In this paper, we focused on a novel proposition of resources reservation mechanism of the traffic submitted to the network with regard to its QoS characteristics by defining a relation between the required constraints of the applications.

Our QoS resources reservation method, QSRR, provides us reserving network path that have sufficient resources to satisfy the QoS requirements of all admitted connections. In this sense, for each flow on which we have provided some QoS guarantee constraints (in term of delay or throughput), QSRR will allocate some resources which will be exclusively for its use. While the resources reservation is based on the available bandwidth, we have use, in QSRR, an efficient method to obtain a relation between the required delay and the required bandwidth.

Finally, simulation analysis have been conducted and produced some performance evaluation results showing that the performances provided by QSRR (in terms of delay, load and throughput) are always better than the ones provided by CSMA.

\section{REFERENCES}

[1] T. Imienlinski and B. R. Badrinath 'Mobile wireless computing: solutions and challenges in data management'. CACM, October 1994.

[2] M. S. Gast and O'Reilly, '802.11 Wireless networks: The definitive guide', 2002.

[3] G. Pujolle, 'Les Réseaux', Troisième Edition, Eyrolles, 2002.

[4] M. Mirhakkak, N. Schult, et D. Thomson. 'Dynamic Quality of Service for Mobile Ad Hoc Networks', Dans IEEE MobiHoc 2000, Boston, Massachusets, USA, Août 2000.

[5] Marie-Ange Remiche, 'ELEC 379 : Réseaux II II', Belgique, 2005.

[6] Hassene Bouhouch et Sihem Guemara El Fatmi, "QoS Routing In Ad Hoc Networks by Integreting Activity in the OLSR Protocol" ICSNC, pp.1, Second International Conference on Systems and Networks Communications (ICSNC 2007), 2007

[7] C. Sarr, C. Chaudet, G. Chelius and I. Guérin Lassous, 'ABE : Un protocole de réservation de bande passante pour les réseaux ad hoc basés sur IEEE 802.11', Technical Report, INSA-Lyon, France, 2008.

[8] A. Johnsson, B. Melander and M. Björkman, 'Bandwidth Measurement in Wireless Network', Technical Report, Mälardalen University, Sweden, 2005.

[9] N. Kettaf, H. Abouaissa, T. Vuduong and P. Lorenz, 'A Cross Layer Control On-demand Routing Protocol for QoS Applications', IJCSNS International Journal of Computer Science and Network Security, Vol 6, N ${ }^{\circ}$ 9B, September 2006. 